\title{
Analysis of motor fan radiated sound and vibration waveform by automatic pattern recognition technique using "Mahalanobis distance"
}

\author{
Eiji Toma ${ }^{1}$
}

Received: 17 October 2017/Accepted: 13 May 2018/Published online: 4 June 2018

(c) The Author(s) 2018

\begin{abstract}
In recent years, as the weight of IT equipment has been reduced, the demand for motor fans for cooling the interior of electronic equipment is on the rise. Sensory test technique by inspectors is the mainstream for quality inspection of motor fans in the field. This sensory test requires a lot of experience to accurately diagnose differences in subtle sounds (sound pressures) of the fans, and the judgment varies depending on the condition of the inspector and the environment. In order to solve these quality problems, development of an analysis method capable of quantitatively and automatically diagnosing the sound/vibration level of a fan is required. In this study, it was clarified that the analysis method applying the MT system based on the waveform information of noise and vibration is more effective than the conventional frequency analysis method for the discrimination diagnosis technology of normal and abnormal items. Furthermore, it was found that due to the automation of the vibration waveform analysis system, there was a factor influencing the discrimination accuracy in relation between the fan installation posture and the vibration waveform.
\end{abstract}

Keywords MT system $\cdot$ Mahalanobis distance (MD) $\cdot$ Feature value $\cdot$ Effectiveness analysis

\section{Introduction}

Demand for compact motor fan for cooling the inside of electronic devices is increasing now due to weight reduction and space saving of IT equipment. In the quality inspection process at the site, quality inspection of compact motor fan is carried out by sensory test of inspector. This sensory test requires a lot of experience in order to accurately diagnose the subtle difference in sound of fan and difference in vibration, and variation in pass/fail judgment occurs due to changes in physical condition and environment of inspector.

In order to solve these quality problems, development of an analysis method capable of quantitatively and automatically diagnosing the sound/vibration level of a fan is required.

Eiji Toma

toma-e@tomakomai-ct.ac.jp

1 National Institute of Technology, Tomakomai College, Tomakomai, Japan
In a previous study, it has been reported on frequency analysis of the acoustic waveform of a compact motor fan and investigating characteristics appearing in normal and abnormal fans. However, it is currently difficult to discriminate and diagnose appropriate normal items/abnormal items from measured values and analysis results obtained by sound and vibration.

In this research, in order to improve the discrimination analysis accuracy of normality/abnormality concerning noise and vibration of motor fan, we worked on analysis research applying waveform information pattern recognition technology.

\section{Research purpose}

In many cases, because noise level of fan depends on the noise level of the whole motor, it is important to reduce the noise. Also, if the fan approaches and interferes with the fan cover on the stationary side, remarkable resonance sound may be generated. The fan noise includes a rotational sound component $(f z)$ which is the product of the 
number of rotations $(f)$ and the number of blades $(z)$ and a turbulent sound component generated by turbulence of the wind flow. Recently, a method of changing the rotation speed by an inverter is used. In this case, with respect to ventilation noise, the rotational sound component $(f z)$ may generate superior noise at a certain rotational frequency.

This outstanding noise is called pure tone and is evaluated as a noisy sound. From studies of conventional fan noise, numerous studies on noise reduction have been made on rotational sound and turbulent sound from flow analysis on the number of blades and optimization of blade shape (Noda 2014).

In precedent research, there was a case of verifying the usefulness by introducing Mahalanobis Taguchi (MT) system which is one of the pattern recognition methods, and understands a thing effective to some extent. In the case of using pattern recognition method, processing for extracting feature value from measurement data is performed. If an invalid feature amount is included in the abnormality detection, sensitivity and reliability of recognition decrease.

Therefore, it is important to set feature values considered valid for discrimination without excess or deficiency. Statistical values such as average value and peak value of the waveform were used as feature value in precedent research. However, in order to improve the accuracy of discrimination, many feature values and number of samples were required. If it is possible to extract feature values effective for abnormality detection from number of few samples, discrimination can be performed with high efficiency.

In this study, we applied a method to extract the feature quantity from the shape information of the waveform proposed in the MT system as the measurement result of the sound and vibration of the motor fan. By evaluating the effectiveness of the obtained feature quantity, we worked on analysis research aimed at improving discrimination accuracy (Teshima et al. 2017).

\section{Analysis principle}

\section{Pattern recognition}

Pattern recognition is a technology to replace information processing such as human judgment and prediction with computer. In other words, it is a process of evaluating how much an unknown input pattern resembles a pre-input standard pattern by numerical values and determining which category it belongs to.

In this research, MT system was adopted as a pattern recognition technology. MT system is an information processing system which constitutes a data group of a reference pattern as a unit space and can judge whether target data belongs to a unit space or not (Teshima et al. 2017).

\section{MT method}

In the MT system, MT method is a method of determining outlier degree from unit space by evaluation index of Mahalanobis distance (MD) and judging whether it is normal or abnormal. The MT method is a method for gaining cognizance of whether the target data (otherwise referred to as unknown data) belongs to the same standard, homogeneous, group. It is an analysis method that defines a homogeneous population as a unit space for purpose and calculates the distance from the unit space center of unknown (target) data as Mahalanobis distance (MD).

The MT method stands on a simple concept and is very easy to use; among the components of the MT system, the MT method has the greatest number of practical applications (Teshima 2012).

As shown in Fig. 1, it is recognized that, if the MD turns out to be short, the pattern is close to the unit space and that, if the MD turns out to be long, the pattern is distant. When the unit space is a normal population, if the MD is short, there is a strong possibility that the target data is normal. MD itself was proposed by Indian mathematician P.C. Mahalanobis and is the result of converting multivariate information in which multiple variables are intertwined complicatedly into distance information (Taguchi 1992; Yano 2011).

\section{Formulation of MD}

Mahalanobis' distance is called MD. It is a kind of distance for multivariable space. Correlation effect is included in this distance (Teshima 2012; Suzuki 2012).

1. Normalize data

MD calculation is possible without normalization. But after normalization, covariance in the next step is

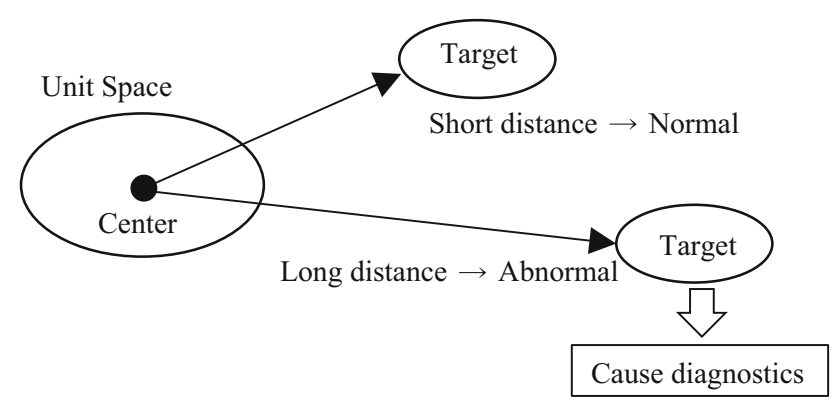

Fig. 1 Conceptual drawing of the MT method 
correlation coefficient. So evaluation of correlation effect is easy.

2. Make covariance matrix

Covariance matrix $=\left(\begin{array}{cc}1 & B \\ B & 1\end{array}\right)$

3. Make inverse matrix of covariance matrix

$$
\begin{aligned}
\left(\begin{array}{ll}
a & b \\
b & a
\end{array}\right) & =\left(\begin{array}{ll}
1 & B \\
B & 1
\end{array}\right)^{-1} \text { or }\left(\begin{array}{ll}
1 & 0 \\
0 & 1
\end{array}\right) \\
& =\left(\begin{array}{ll}
a & b \\
b & a
\end{array}\right)\left(\begin{array}{cc}
1 & B \\
B & 1
\end{array}\right)
\end{aligned}
$$

4. Calculate the square of MD

$$
\mathrm{MD}_{i}^{2}=\left(x_{1 i}, x_{2 i}\right)\left(\begin{array}{cc}
a & b \\
b & a
\end{array}\right)\left(\begin{array}{c}
x_{1 i} \\
x_{2 i}
\end{array}\right)
$$

$\mathrm{MD}^{2}=X A X($ If $A$ is inverse matrix and $X$ is data $)$

\section{MD derivation and certification}

The concept of MD is a value obtained by consolidating the deviation from the relationship between the distance and the correlation from the average of the object of interest in consideration of the correlation of the plurality of variables (Suzuki 2012).

MD in the relation of the target data of two items is given by following expression.

$\mathrm{MD}=\sqrt{\frac{u_{1}^{2}-2 r u_{1} u_{2}+u_{2}^{2}}{1-r^{2}}}$

$u_{1}=\frac{x_{1}-m_{1}}{\sigma_{1}}$

$u_{2}=\frac{x_{2}-m_{2}}{\sigma_{2}}$

$\left\{\begin{array}{l}x_{1}, x_{2}: \text { Target data } \\ m: \text { Average } \\ \sigma: \text { Standard deviation } \\ r: \text { Correlation coefficient }\end{array}\right.$

Equations (1)-(3) mean to investigate how many times the distance from the average corresponds to the standard deviation which is an index of the variation of all data. The MD calculation formula obtained by expanding $\sqrt{ }$ in Expression (1) to a plurality of items is as follows: $\left(u_{1} \ldots u_{k}\right)$ on the right side is a value normalized by using the average and standard deviation of subscript item data.
$\mathrm{MD}=D^{2}=\left(u_{1} \cdots u_{k}\right)\left[\begin{array}{ccc}1 & \cdots & r_{1 k} \\ \vdots & \ddots & \vdots \\ r_{k 1} & \cdots & 1\end{array}\right]^{-1}\left[\begin{array}{c}u_{1} \\ \vdots \\ u_{k}\end{array}\right]$

$u_{k}=\frac{x_{k}-m_{k}}{\sigma_{k}}$

$m_{k}:$ Average of data group

$\sigma_{k}:$ Standard deviation of data group

$\left[\begin{array}{ccc}1 & \cdots & r_{1 k} \\ \vdots & \ddots & \vdots \\ r_{k 1} & \cdots & 1\end{array}\right]^{-1}$

It means the inverse matrix of the correlation matrix.

When Eq. (4) is verified in the case of $k=2$,

$\mathrm{MD}=D^{2}=\left(u_{1} u_{2}\right)\left(\begin{array}{ll}1 & r \\ r & 1\end{array}\right)^{-1}\left(\begin{array}{l}u_{1} \\ u_{2}\end{array}\right)$

The inverse matrix equation of the $2 \times 2$ matrix is,

$$
\begin{aligned}
& \left(\begin{array}{ll}
a & b \\
c & d
\end{array}\right)^{-1}=\frac{1}{a \cdot d-b \cdot c}\left(\begin{array}{cc}
d & -b \\
-c & a
\end{array}\right) \\
& \left(\begin{array}{ll}
1 & r \\
r & 1
\end{array}\right)^{-1}=\frac{1}{1-r^{2}}\left(\begin{array}{cc}
1 & -r \\
-r & 1
\end{array}\right)
\end{aligned}
$$

Therefore, the following expression is obtained,

$$
\begin{aligned}
\therefore D^{2} & =\left(u_{1} u_{2}\right) \frac{1}{1-r^{2}}\left(\begin{array}{cc}
1 & -r \\
-r & 1
\end{array}\right)\left(\begin{array}{l}
u_{1} \\
u_{2}
\end{array}\right) \\
& =\frac{1}{1-r^{2}}\left(u_{1}-r u_{2}-r u_{1}+u_{2}\right)\left(\begin{array}{l}
u_{1} \\
u_{2}
\end{array}\right) \\
& =\frac{u_{1}^{2}-2 r u_{1} u_{2}+u_{2}^{2}}{1-r^{2}}
\end{aligned}
$$

It proved to satisfy the expression in $\sqrt{ }$ Eq. (1).

In the MT method, in order to normalize the average of the squared values of MD to 1 , an operation of dividing the value of $\mathrm{MD}=D^{2}$ by the number of items $k$ is added. Therefore, the calculation formula of MD used in the MT method is as follows.

$\therefore D=\sqrt{\mathrm{MD}}=\sqrt{\frac{1}{k}\left(u_{1} \cdots u_{k}\right)\left[\begin{array}{ccc}1 & \cdots & r_{1 k} \\ \vdots & \ddots & \vdots \\ r_{k 1} & \cdots & 1\end{array}\right]^{-1}\left[\begin{array}{c}u_{1} \\ \vdots \\ u_{k}\end{array}\right]}$

\section{Discrimination method}

With the MT method, normality/abnormality is determined by comparing MD of target data with the threshold value. When the MD is small close to 1, it is judged that it belongs to the unit space, and if MD is larger than the set threshold 
value, it is judged not to belong to the unit space. The judgment threshold with the MT method is generally said to boil down to 4 or thereabouts. This is because, for all practical statistical mathematics purposes, if the MD exceeds 4 , the probability of unknown (target) data being a member of the unit space shrinks to a small possibility (Wakui 2014).

However, it is currently reported that the method of setting a threshold using the $\chi^{2}$ distribution is highly effective. For this reason, we set the threshold using the $\chi^{2}$ distribution in this research.

In Fig. 2, the abscissa is the Mahalanobis distance, the ordinate is the probability, and the graph curve $\left(\chi^{2}\right.$ distribution theoretical curve) means the probability that the square value of the Mahalanobis distance takes (Yano 2004).

\section{Feature extraction}

The features of waveforms include frequency and amplitude, and categories such as average frequency, magnitude of oscillation, and maximum magnitude of oscillation have been commonly used. Frequency analysis (FFT: Fast Fourier Transform) and wavelet have also been used. Frequency analysis is a technique of expressing the characteristics of waveforms in terms of a frequency axis and an energy axis. A wavelet is a technique of expressing the characteristics of waveforms in terms of a time axis, in addition to the frequency axis and the energy axis.

Frequency analysis and wavelets are excellent methods of explaining the characteristics of waveforms. Nonetheless, it cannot quite be said that both convey sufficient information on the characteristics of the given waveform pattern.

For instance, frequency analysis is a processing method dealing with waveforms over a relatively long period of time and is therefore not adept at capturing waveform

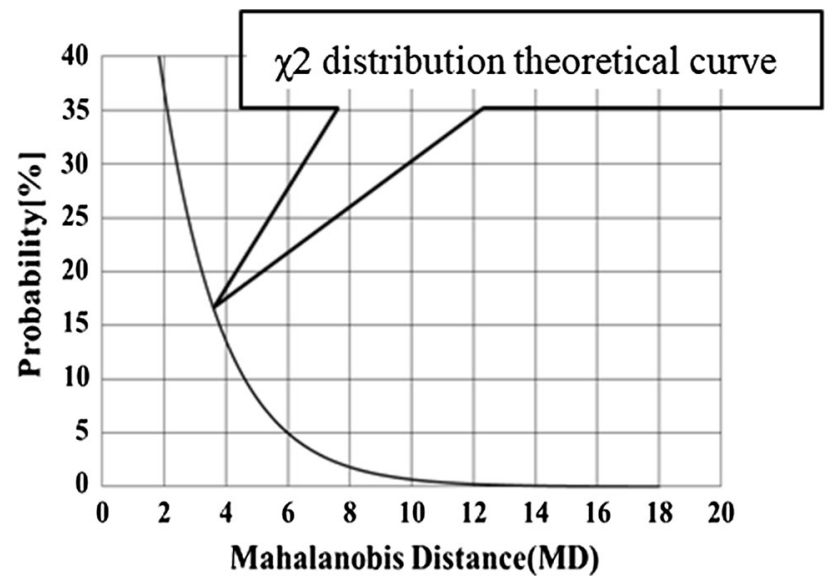

Fig. 2 Distribution of squared values of MD changes that may be occurring within short time spans. Furthermore, both frequency analysis and wavelets presuppose the human operator's evaluation and judgment of the results they produce.

The variation and abundance information extraction method was proposed as a means of expressing the characteristics of waveform patterns in more accurately quantified terms.

In this research, variation and abundance information is extracted from the radiated sound waveform from compact fan motor, and the waveform characterized. First, a parallel line (reference line) is drawn on the time axis at a constant interval in the waveform in the time domain, and a section on the time axis (extraction width) for calculating the characteristic amount is set. Next, the number of times of intersection with the reference line within interval of extraction width is taken as the variation, and sum of the values in the range above the reference line is obtained as the abundance information. Performing calculation of this variation and abundance information in all sections of the waveform data is called "waveform analysis."

Figure 3 shows the concept of variation and abundance information (differential and integral characteristics) in feature extraction (Yano 2004; Teshima 2012).

\section{Experimental system of radiated sound}

\section{Noise level of the test fan}

As shown in Fig. 4, fan motors generally rotate in both directions, so that the blades of the fan use radial blade (same type as the test fan). Radial blades have low cooling efficiency and high noise. The noise level is obtained from Eq. (7). However, the evaluation position of the noise is the axial center height of the motor, and the distance is $1 \mathrm{~m}$. (Noda 2014).

Noise level; $A[d b]=70 \log D+50 \log N+k$

$$
\begin{aligned}
& D: \text { Outer diameter of the blades }[m] \\
& N: \text { number of rotations }[r p s] \\
& k: \text { constan } t(=32 \sim 36)
\end{aligned}
$$

From Eq. (7), it is important to reduce the outer diameter $D$ of the fan in order to lower the noise level. However, since the wind volume and the wind pressure also decrease, the balance between these and the noise becomes important at the time of designing.

That is, the relation of Expression (8) is obtained.

Wind volume $=\pi \varphi U D B$

Here, $\varphi$ : flow coefficient $(0.15-0.25), U$ : outer peripheral speed of the blades, and $B$ : width of the outer 


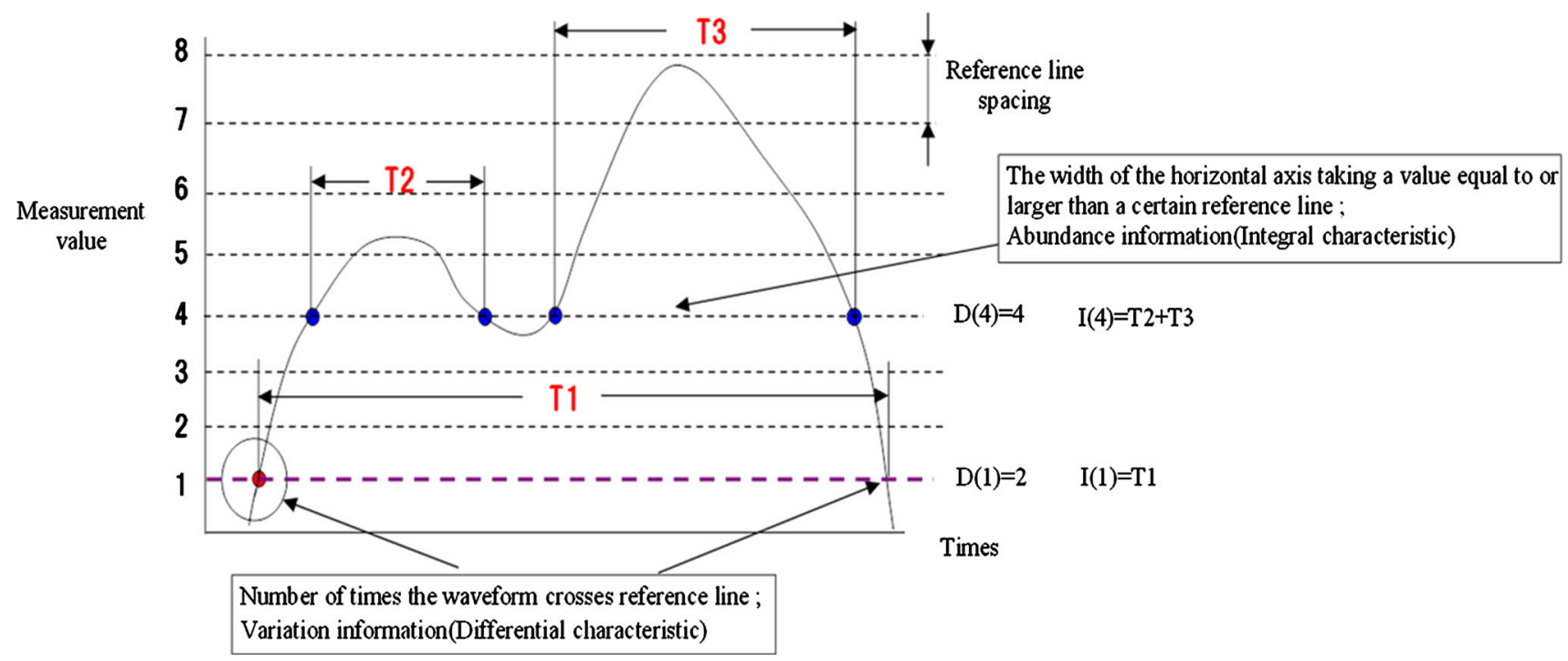

Fig. 3 Variation and abundance information

Fig. 4 Fan shape
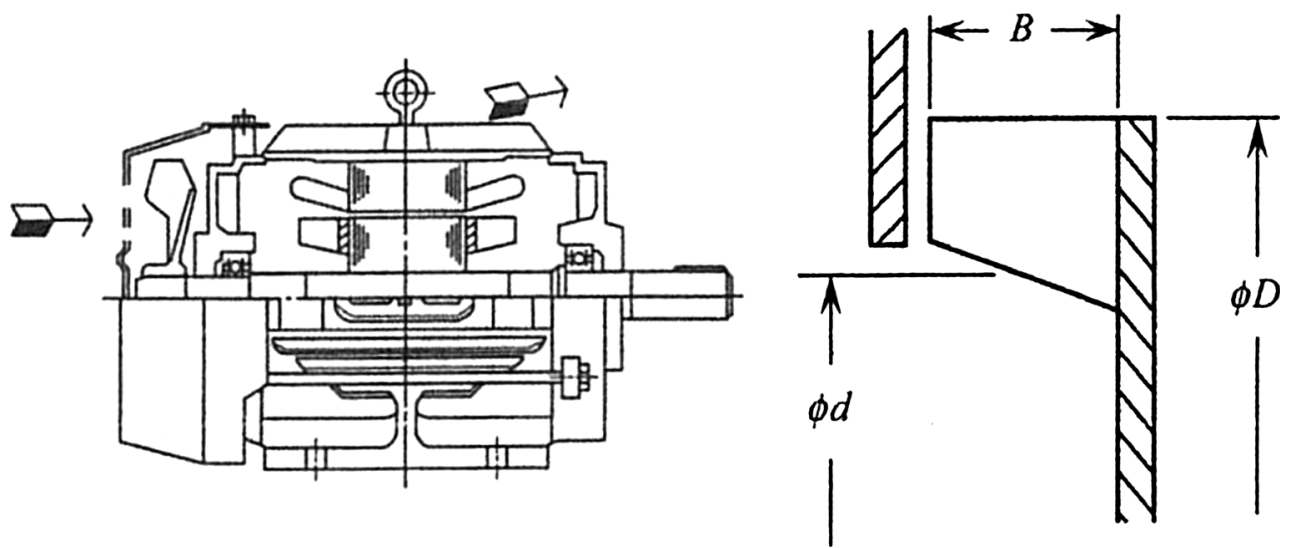

peripheral portion of the blades. Attention is required since the air volume decreases with the square of $D$.

\section{Test fan}

In this research, we used two kinds of compact fan motors. Sample items shown in Fig. 5 (and Table 1) are five

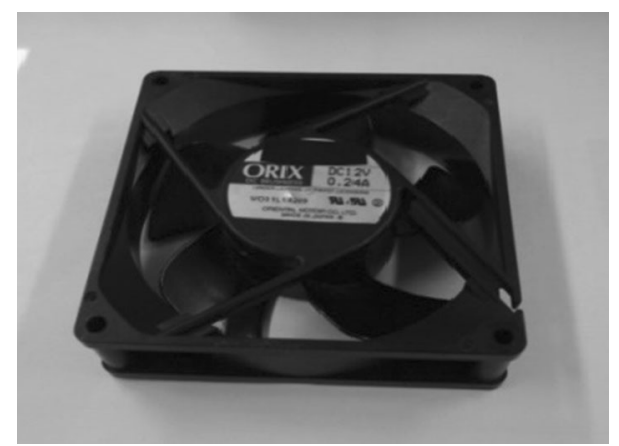

Fig. 5 Test fan
Table 1 Specification of fan

\begin{tabular}{ll}
\hline Fan flame size & $92.5 \times 92.5 \times 25.4(\mathrm{~mm})$ \\
Blade number & 5 \\
Rated voltage & DC12 V \\
Rotational speed & $3400(\mathrm{rpm})$ \\
\hline
\end{tabular}

normal products (\#9701-\#9705), and sample items shown in Fig. 6 (and Table 2) including 10 normal products (fan 1 to fan 10) are five abnormal products (fan \#1-fan \#5). The abnormal product has surface buckle, and each displacement is $14 \mu \mathrm{m}$ for fan \#1, $25 \mu \mathrm{m}$ for fan \#2, $50 \mu \mathrm{m}$ for fan $\# 3,70 \mu \mathrm{m}$ for fan \#4, and $90 \mu \mathrm{m}$ for fan \#5. Surface buckle refers to the size when the end face rotating around a certain axis deviates from a plane perpendicular to this axis during rotation. 


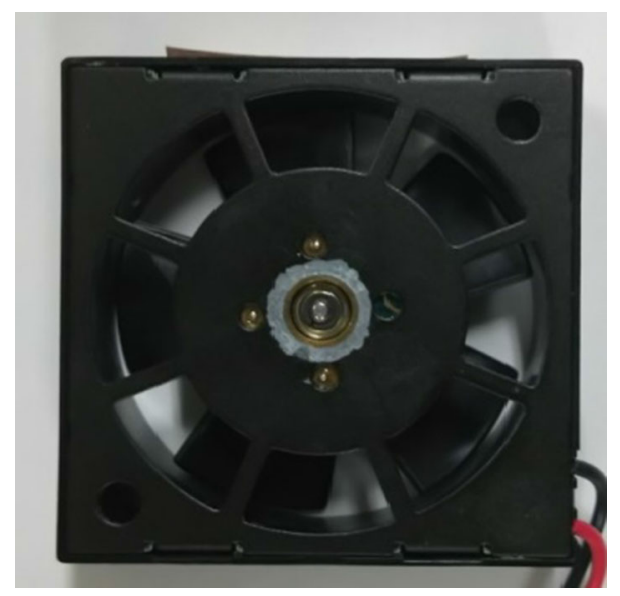

Fig. 6 Test fan

Table 2 Specification of fan

Fan flame size

$42 \times 42 \times 10(\mathrm{~mm})$

Blade number

Rated voltage

DC24 V

Rotational speed

$10,000(\mathrm{rpm})$

\section{Measurement of radiated sound}

Measurement of the sound of compact fan motor was taken in a quiet room. A schematic diagram of recording is shown in Fig. 7.

The sound collection was set to $30 \mathrm{~mm}$ above the rotation axis center from the rotation surface on the fan suction side by a $1 / 2$ inch condenser microphone. The

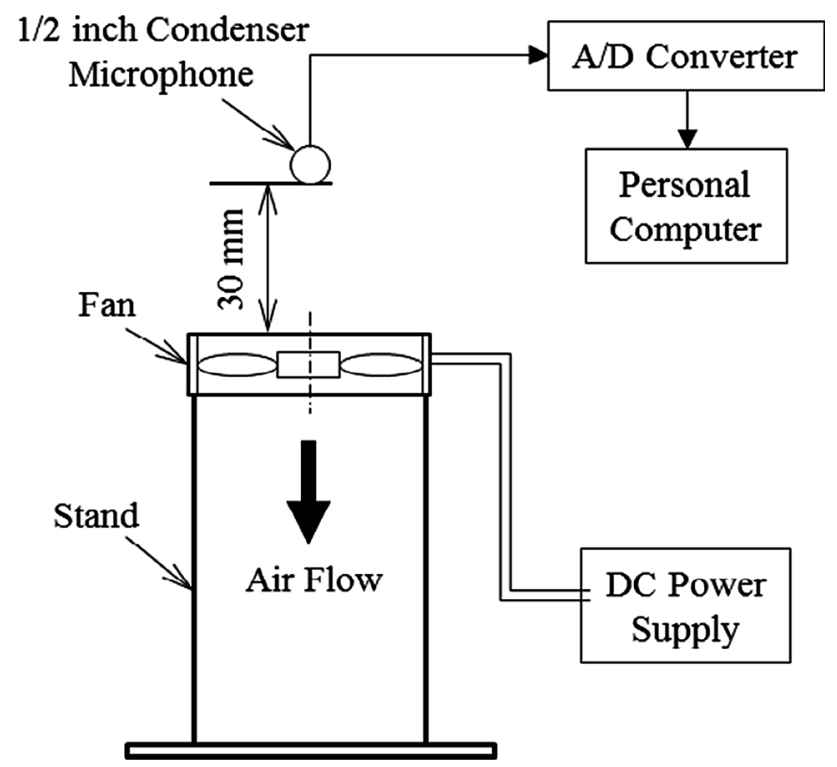

Fig. 7 Recording system reason is based on the distance between ear and fan when inspector performs sensory test. Fan recording time was $10 \mathrm{~s}$, sampling frequency was $51.2[\mathrm{kHz}]$, and signal from microphone was saved in $\mathrm{PC}$ via A/D converter. Figure 8 shows the waveform data obtained by measuring the radiated sound of fan. Measurement sample number and time of fan's radiated sound is " 512,000 samples $=10 \mathrm{~s}$ " at sampling frequency $51.2[\mathrm{kHz}]$. However, it was judged that a more accurate analysis could be performed by capturing a behavior change for a short time, and waveform data of "5120 samples $=0.1 \mathrm{~s}$ " were analyzed.

\section{Analysis procedure}

The analysis procedure of radiated sound and vibration waveform is as follows.

1. Feature extraction

In order to characterize the waveform, extraction width and reference line set, and variation and abundance values are extracted as the feature value.

2. Creating unit space

Although the unit space consists of a plurality of samples and their variables (feature items), MT method has a condition that the number of samples in unit space must be larger than the number of variables, and more than 3 times the variable is considered ideal.

3. Setting a threshold

The threshold was determined with a probability of $5 \%$ according to the $\chi^{2}$ distribution. This value is considered to be the boundary value when judging things in statistics, and it is called the significance level.

4. MD calculation of target data

The waveform analysis of the abnormal fan and feature value of unit space are extracted, and the MD value is calculated by Eq. (6).

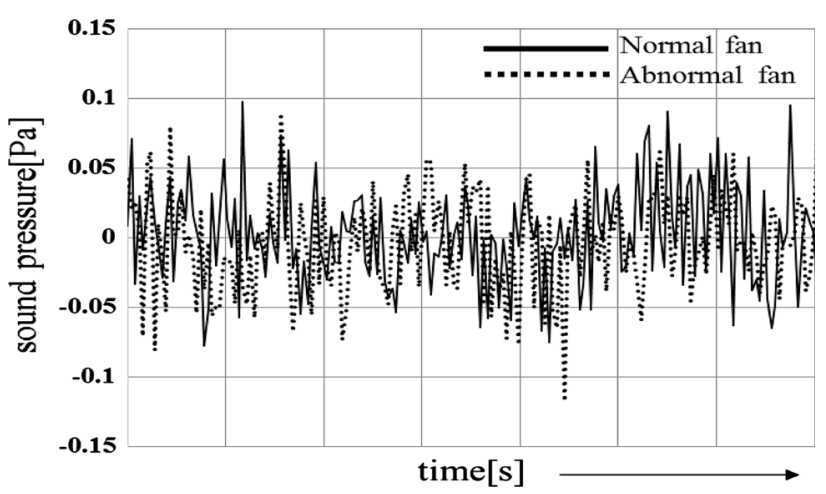

Fig. 8 Waveform data 
5. Effectiveness analysis

Effectiveness analysis is a process of evaluating which item is effective for abnormality detection by $\mathrm{SN}$ ratio.

6. Evaluation of effectiveness by $\mathrm{SN}$ ratio

The MT system includes a success/failure quantification proposal for the recognition system.

Use is made here of the concept of SN ratio (signal-tonoise ratio), which provides a functionality evaluation yardstick in quality engineering or MT method. In pattern recognition, generally speaking, the recognition result is influenced by various factors, ranging from measuring tool selection to recognition software parameter settings. Use of the $\mathrm{SN}$ ratio makes it possible to evaluate the appropriateness of the entire recognition system.

In quality engineering there are concepts of various SN ratios, but there are the following two methods for calculating the SN ratio used in the MT system.

(a) If it is difficult to give a numerical value to the degree of abnormality, apply the $\mathrm{SN}$ ratio of the preferably large characteristics.

(b) When the degree of abnormality can be quantified, apply the $\mathrm{SN}$ ratio of the dynamic characteristic.

It is desirable that the MD value of data known not to belong to the unit space be as large as possible. In this research, "SN ratio of the preferably large characteristics" was applied.

In quality engineering, "larger preferable characteristics" are called preferably large characteristics. Since there are generally variations when there are many data, the SN ratio is to quantify desirability collectively including variations.

The calculation formula of $\mathrm{SN}$ ratio $(\eta)$ of preferably large characteristic is as shown in Eq. (9). At this time, it is better that the $\mathrm{SN}$ ratio is large.

Here, $D^{2}$ is the Mahalanobis distance (the value of the square), and $k$ is the number of signal data not belonging to the unit space.

$\eta=-10 \log \left\{\left(1 / D_{1}^{2}+1 / D_{2}^{2}+\cdots+1 / D_{k}^{2}\right) / k\right\}$

\section{Analysis result of radiated sound}

\section{Analysis result of normal products}

First, we analyzed a waveform in the fan of Fig. 5 and extracted feature value. The fixed value is extraction width 20, reference line 10 . Therefore, 20 samples can be obtained from one waveform. In addition, since the reference line calculates the variation and abundance information for each extraction width, the number of variables is
20 pieces. Next, taking these into consideration, three waveforms were arbitrarily selected from five normal products (\#9701-\#9705) so as to be three times the number of variables, and a unit space was created. Finally, waveform analysis was also performed on normal products not belonging to the unit space, and the MD value was calculated. Figure 9 shows the calculation result of the MD value of the normal product. On the graph, it is judged to be abnormal because the MD value exceeding the threshold can be confirmed. However, it is understood that because the normal product is the target, it is not an appropriate analysis result. It is estimated that variables invalid in discrimination are included in feature values. Therefore, it is necessary to analyze and evaluate which items are highly effective. Figure 10 shows the results of the effectiveness analysis on the target data. The horizontal axis represents the variable, and vertical axis represents the $\mathrm{SN}$ ratio, which means that the item with the highest value is valid for the judgment. Therefore, Fig. 11 shows the result of calculating the MD value using the variable with the positive $\mathrm{SN}$ ratio as the feature value. On the graph, it is judged that the MD value is equal to or less than the threshold value and it is judged to be normal, and it is judged that the effective feature value can be extracted.

\section{Analysis result of abnormal products}

Next, we analyzed a waveform in a fan of Fig. 6 and extracted feature value. The fixed value is extraction width 20 and reference line 30 . The number of variables is 60 . In the unit space, nine waveforms were arbitrarily selected from 10 normal products (fan 1 to fan 10) so that the number of samples was three times as large as the variable. Waveform analysis was performed on abnormal products (\#fan 1-\#fan 5). Figure 12 shows MD values of normal and abnormal items not belonging to the unit space.

From the results, MD values above the threshold were shown for all abnormal items, and it was determined as abnormal. However, among the normal products, items

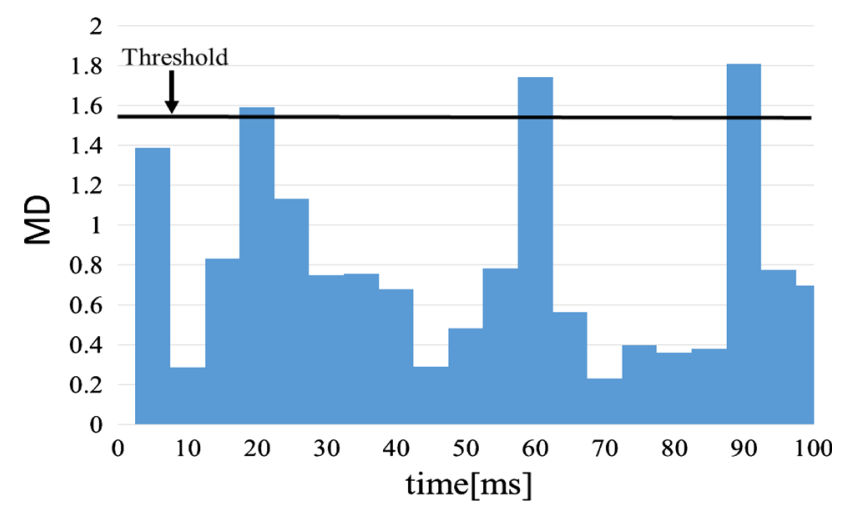

Fig. 9 MD calculation result of normal products 


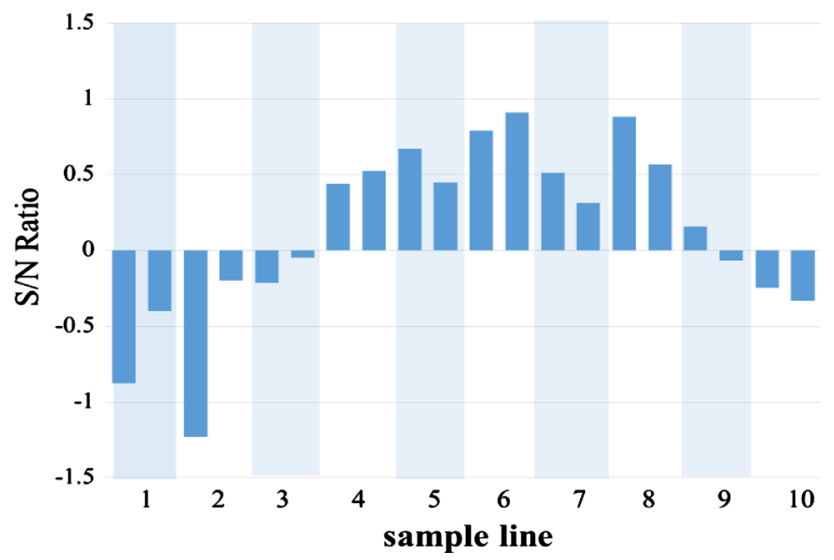

Fig. 10 Effectiveness analysis result

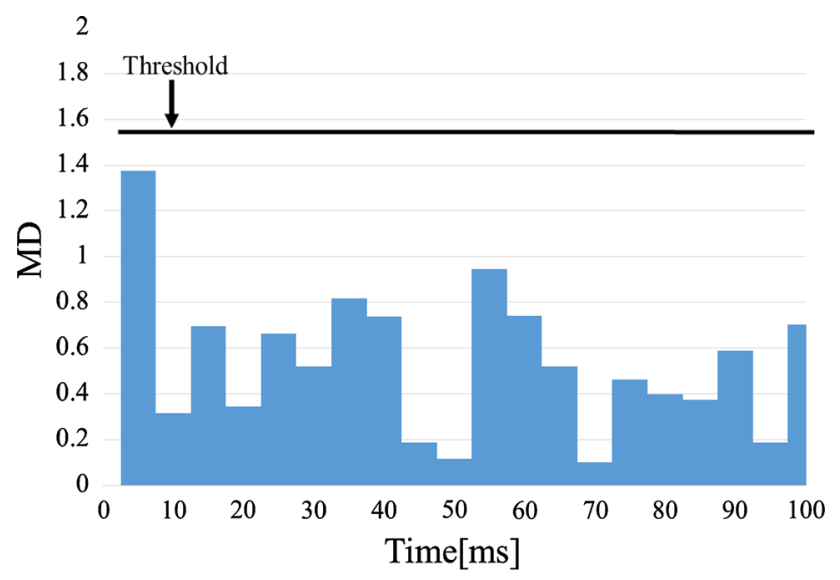

Fig. $11 \mathrm{MD}$ calculation result after analysis

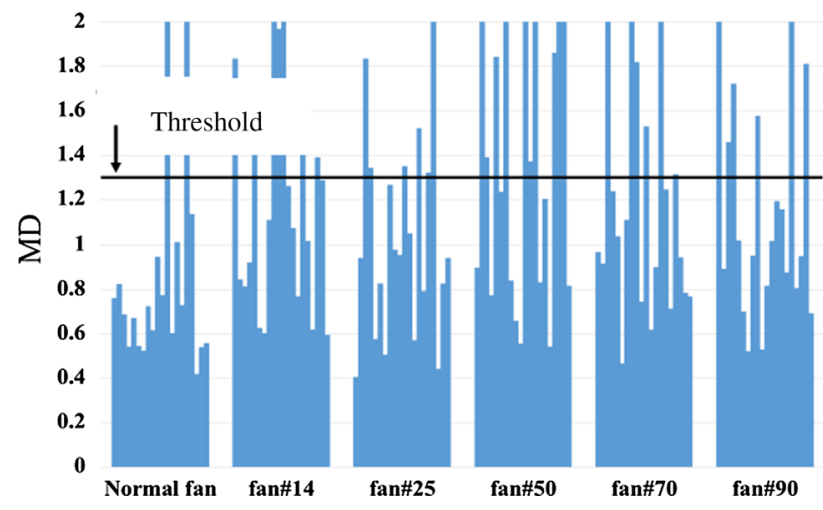

Fig. $12 \mathrm{MD}$ calculation result of each sample fan

which are judged to be abnormal items beyond the threshold were confirmed. Figure 13 shows the effectiveness analysis result of the waveform of the abnormal product (\#fan 1). From the figure, the SN ratio shows a positive value in the central part, and all values are negative at both ends.

The same tendency was shown also in the effectiveness analysis of other abnormal items. A variable taking a

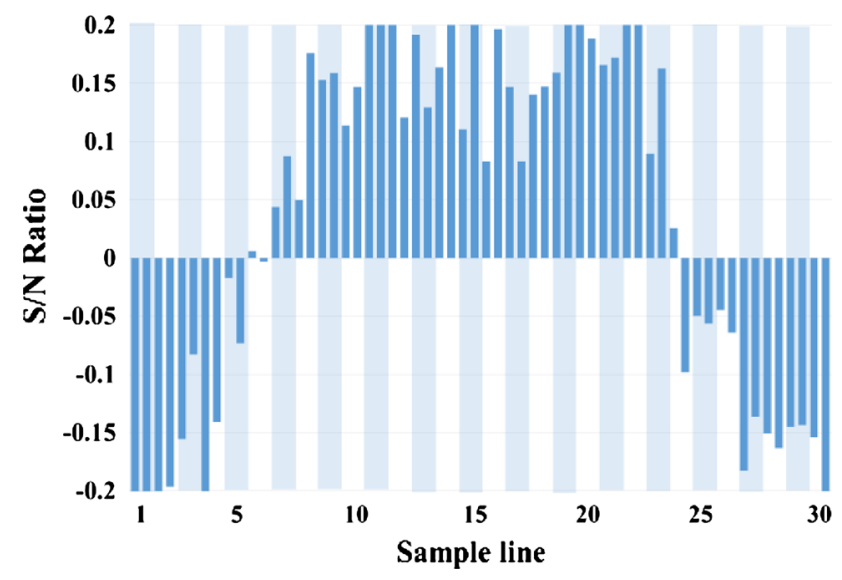

Fig. 13 Effectiveness analysis result

negative value is variation and abundance information of reference line. By using variation and abundance information of reference line at the central portion of the waveform as the feature value, it is shown that accurate abnormality determination is possible.

Figure 14 shows the result of calculating the MD value using the variable whose $\mathrm{SN}$ ratio is a positive value as the feature quantity from the result of effectiveness analysis of all abnormal items. From the results in the figure, it is found that the normal value is equal to or less than the threshold value and the abnormal item is the MD value that is equal to or more than the threshold value, so it is an appropriate discrimination.

\section{Experiment system of vibration}

\section{Vibration measurement}

When the motor fan is sensory-tested, the inspector holds two positions on the side of the fan frame and diagnoses by hearing and tactile sense near the ear while turning the wrist.

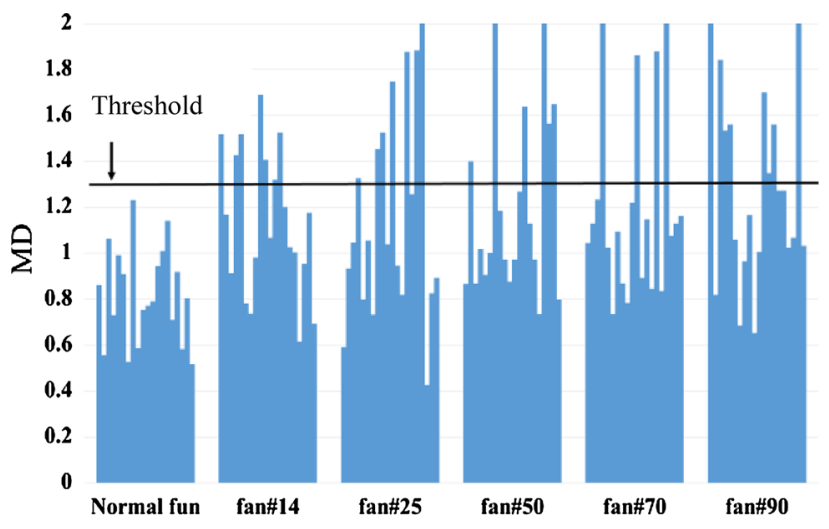

Fig. 14 MD calculation result of each sample fan 
In this study, we developed an automatic rotary oscillating device simulating the handling performed by the inspector and analyzed the vibration waveform pattern by automatic detection of vibration of the motor fan by applying the MT method. Figures 15 and 16 show the automatic rotary oscillating device. As the vibration measurement condition, the rotation speed of the servo motor was set to $3 \mathrm{rpm}$, the fan rotation speed was $3400 \mathrm{rpm}$, and the measurement time was $40 \mathrm{~s}$. In addition, angle of oscillation $\theta$ of the fan was measured in the axial direction and the radial direction under three conditions of $15^{\circ}, 30^{\circ}$, and $45^{\circ}$.

\section{Waveform data of vibration}

The waveform data of vibration were measured using a level recorder, but the test fan of Fig. 6 had a low vibration level and could not obtain sufficient waveform data. Therefore, in this research, the test fan of Fig. 5 was adopted. \#9701, \#9702, \#9703, \#9704, and \#9705 of samples with different magnitudes of vibrations were set in order of increasing vibration acceleration level. Figures 17, 18 show the vibration waveform data in the axial direction and the radial direction with the rotary oscillating device with the angle of oscillation of the fan set to $\theta=15^{\circ}$.

In the vibration waveform in the axial direction of Fig. 17, it can be confirmed that the vibration acceleration level increases in order of the nominal number. On the other hand, since the waveform behavior in the radial direction other than \#9705 is very small, it is difficult to judge the magnitude of the vibration level from the waveform. Furthermore, the same tendency was observed under different angle of oscillation conditions.

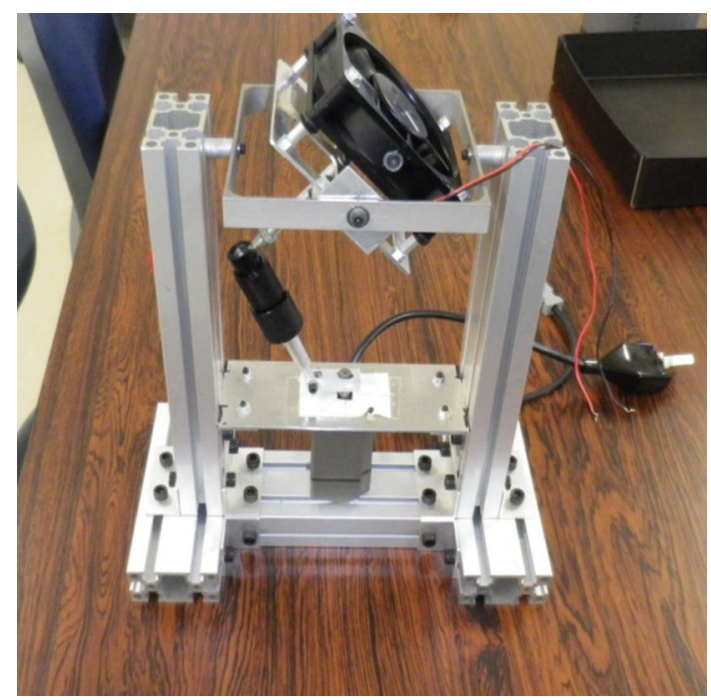

Fig. 15 Overview of automatic rotary oscillating device

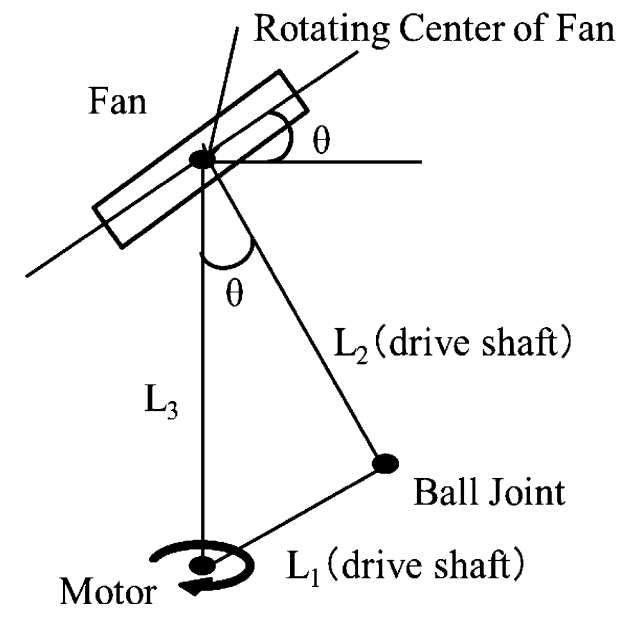

Fig. 16 Schematic of automatic rotary oscillating device

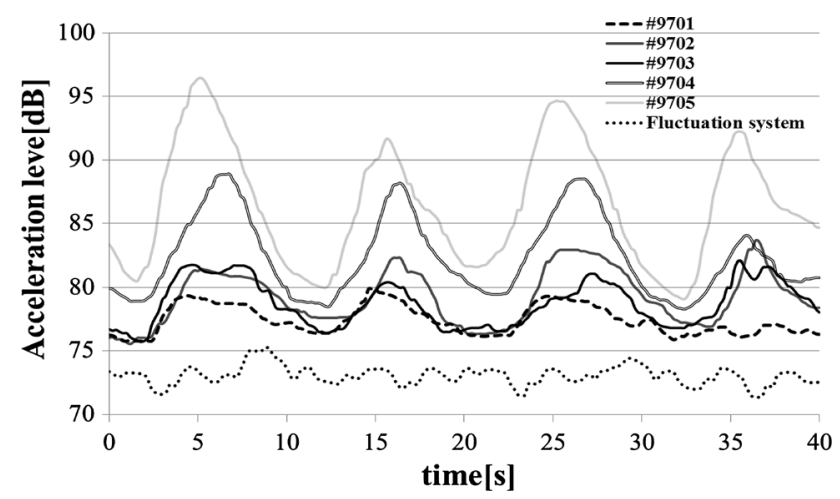

Fig. 17 Acceleration level of axial direction with angle of oscillation $15^{\circ}$

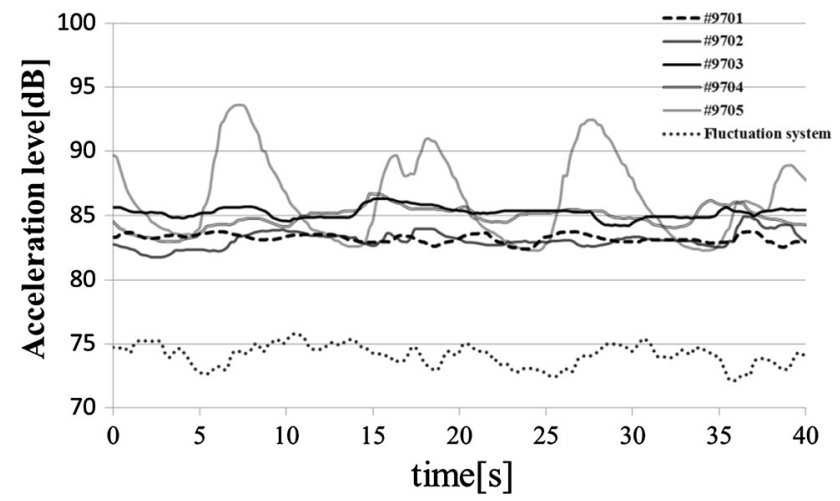

Fig. 18 Acceleration level of radial direction with angle of oscillation $15^{\circ}$

Therefore, in this study, waveform analysis was performed on the axial vibration waveforms of three conditions of the fan's swinging angles of $15^{\circ}, 30^{\circ}$, and $45^{\circ}$ in the same way as the analysis procedure of radiated sound. 


\section{Analysis result of vibration}

The setting value of the waveform analysis in the vibration waveform of the test fan shown in Fig. 5 was set to extraction width 20 and reference line 10 . Since the number of variables is 20, three waveforms are arbitrarily selected from five normal products (\#9701-\#9705) so as to make the number of samples three times as large, and a unit space was created. Waveform analysis was also performed on normal goods not belonging to the unit space to calculate the MD value.

In this study, we analyzed vibration waveforms in the axial direction of three conditions of fan's angle of oscillation of $15^{\circ}, 30^{\circ}$ and $45^{\circ}$, and Fig. 19 shows the result of MD value calculated for each angle of oscillation of the fan. From Fig. 19, it is determined that the MD value is over the threshold value when angles of oscillation are $15^{\circ}$ and $30^{\circ}$, so it is determined to be abnormal. Even at angle of oscillation of $45^{\circ}$, although there are places where the MD value is larger than the threshold value, the best result among the three conditions was obtained.

Figure 20 shows the results of effectiveness analysis for each angle of oscillation. In the analysis results of angles of oscillation of $15^{\circ}$ and $30^{\circ}$, it is considered that the fact that the number of variables in which the $\mathrm{SN}$ ratio becomes negative is large is a factor that increases the MD value. On the other hand, since the number of variables in which the $\mathrm{SN}$ ratio becomes negative at angle of oscillation of $45^{\circ}$ is small, it can be judged that feature quantities effective for discrimination can be extracted.

Figure 21 shows the result of calculating the MD value with the variable whose $\mathrm{SN}$ ratio at angle of oscillation of $45^{\circ}$ is positive as the feature quantity. From the results in Fig. 21, it can be judged that an appropriate discrimination result was obtained because the MD value is below the threshold value.

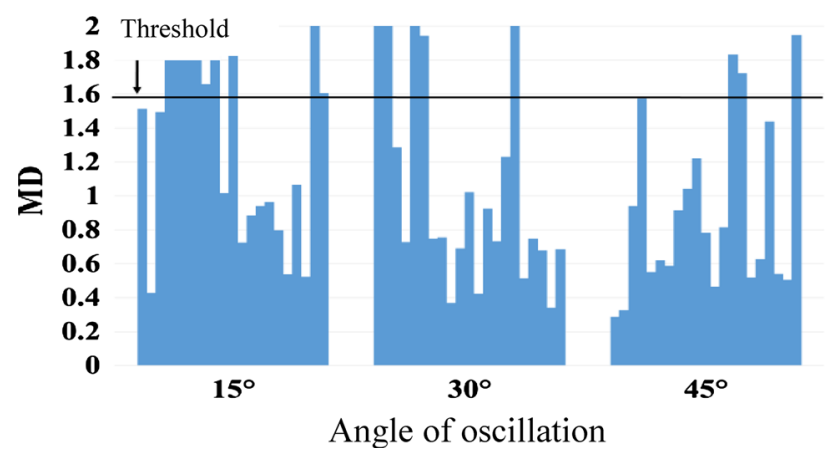

Fig. 19 MD calculation result of each angle of oscillation

\section{Conclusion}

\section{Method of pattern recognition}

Processing to recognize, understand, and predict based on various information obtained from objects is called pattern recognition. Technology replaces information processing such as human judgment and prediction with computer. Although there are familiar practical examples such as character recognition and fingerprint authentication, it is still a big technical subject for computers. The MT system adopted in this research is a system of new pattern recognition and prediction technology proposed by Dr. Taguchi Genichi (Taguchi 2008; Tamura 2009).

Although the application field of pattern recognition is diverse, we are expected to apply it in the whole human activity field of human beings. In particular, it is thought that it plays an important role in terms of accumulation and reproduction of expertise, mainly in manufacturing and medical care. The procedure for carrying out pattern recognition can be roughly divided into three stages. The first step is preparatory preparation, the second step is from measurement to feature extraction, and the third step is recognition processing (Tamura 2009).

\section{Reasons selected}

In the MT system, variation information (differential characteristic) and abundance information (integration characteristic) are proposed as a feature extraction technique. These feature quantities are highly versatile and can be applied to many technical problems. Also, various measurement values often draw waveform patterns. Of course, not only the vibration but also the measured values that change in time series are all waveform patterns. The technique of extracting the features of the waveform as numerical values can be said to be an important technique for problems such as inspection and monitoring (Hasegawa 2007; Taguchi 2002).

\section{Research results}

In this study, we applied the method of extracting the feature quantity from the shape information of the waveform proposed in the MT system for the measurement result of the sound and vibration of the motor fan. Then, we evaluated the effectiveness of the feature value of the acquired waveform information from the MD value and carried out analytical research aimed at improving the detection accuracy of the discrimination. Ultimately, in waveform analysis of radiated sound and vibration of the motor fan, appropriate judgment results of normal and 
Fig. 20 Effectiveness analysis result

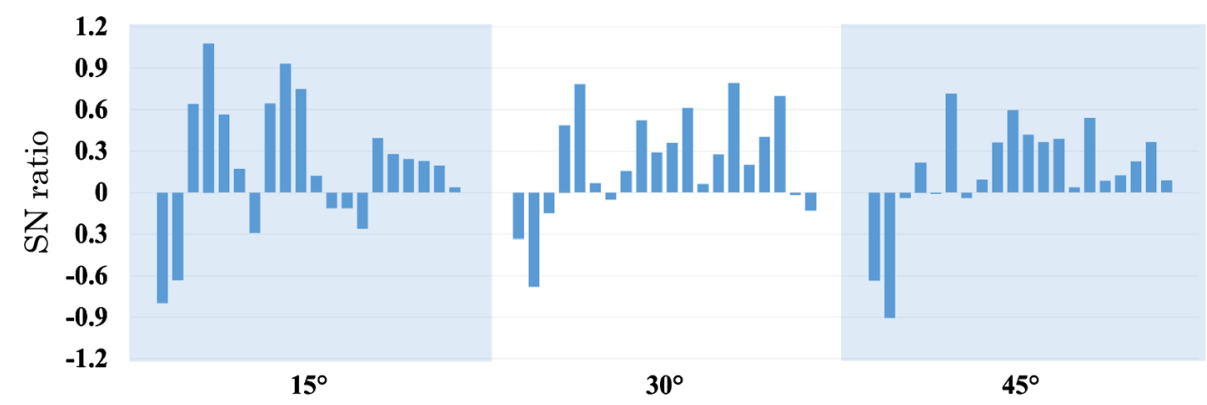

Angle of oscillation

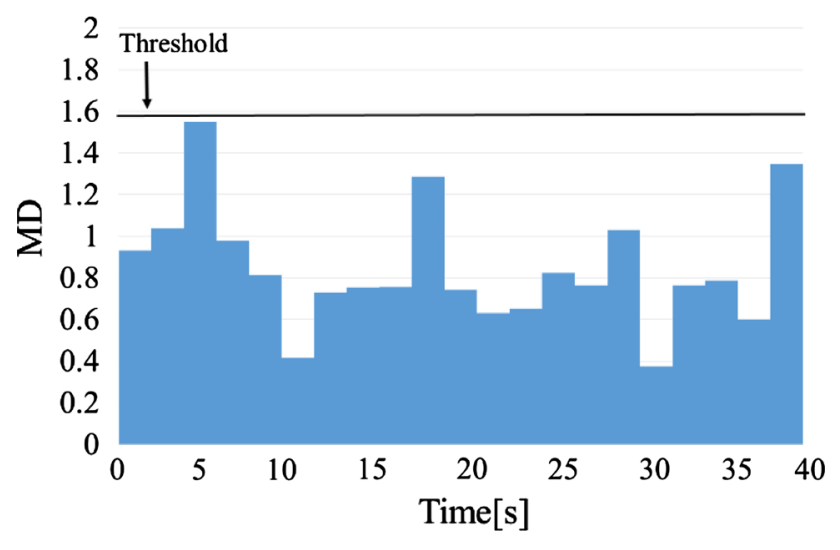

Fig. 21 MD calculation result of angle of oscillation

abnormal items were obtained by extracting feature values effective for detection by effectiveness analysis.

A general pattern recognition method is said to be able to accurately classify abnormal patterns if many data of assumed abnormal patterns can be collected. However, in reality, normal data can be collected, but abnormal data collection becomes more difficult as the scale of the facility becomes more complicated. Since the MT method evaluates the measurement data with the difference from the normal data group as the reference, data collection for learning only needs to be normal data, and this method can cope with an unknown abnormal pattern.

In general, frequency analysis (spectral analysis, fast Fourier transform analysis, etc.) and wavelet are adopted as a characterization analysis method from waveform information (Yano 2002; Mori 2005).

The differences between these analysis methods and variation and abundance information, which are feature values in the MT method, are summarized below.

1. Because of the frequency analysis and wavelet, the nature and homogeneity of the waveform are displayed by graphs and figures, so it is an expression understood by humans.

2. Since variation and abundance information is numerical information expressing the waveform feature, it is difficult to grasp the properties of the waveform by merely looking at the information by a person. However, since it is information that can be directly given to a computer, it can be used as it is for pattern recognition.

3. Since frequency analysis shows the average property of a sufficiently long waveform, it is difficult to capture the change occurring in a short time. On the other hand, feature extraction by variation and abundance information extracts feature values every fixed time width, so it is possible to detect a pattern difference in units of time width (Teshima 2012).

In addition, from the analysis result of the vibration waveform of the present study in the MD value, it was found that the fluctuation of the vibration width of the waveform tends to become small as the angle of oscillation increases.

Regarding future research activities, we will focus on the correlation between angle of oscillation and width by application of MT method in discriminant analysis of normality/abnormality from motor fan vibration waveform and plan to analyze more specific factors is there.

Open Access This article is distributed under the terms of the Creative Commons Attribution 4.0 International License (http://creative commons.org/licenses/by/4.0/), which permits unrestricted use, distribution, and reproduction in any medium, provided you give appropriate credit to the original author(s) and the source, provide a link to the Creative Commons license, and indicate if changes were made.

\section{References}

Hasegawa Y (2007) Story of MT System, Japan Science and Technology Unit, pp 6-11

Mori T (2005) Application and mathematics of the Taguchi methods. In: Optimization engineering using the Taguchi methods, trendy book, Mori Engineer Office, Shizuoka, pp 323-338, 511-583

Noda S (2014) Noise and vibration of motor and countermeasure design method, Scientific Information Publication, pp 19-32, $103-123$

Suzuki M (2012) Introduction to MT system analysis method, Nikkan Kogyo Shimbun, pp 7-101 
Taguchi G (1992) Quality engineering lecture no. 5, Quality engineering casebook-Japan public, Japan Standards Association, pp 1-12

Taguchi G (2002) Evaluation technology for optimization design, Japan Standards Association, pp 189-200

Taguchi G (2008) Technology development in MT system, Japan Standards Association, pp 27-37

Tamura K (2009) New technology of pattern recognition by quality engineering, Japan Standards Association, pp 26-85

Teshima S (2012) Introductory MT system. Japan Science and Technology Publishing, Tokyo, pp 33-57
Teshima S, Tamura K, Hasegawa Y (2017) Standardization and Quality Control 2017. J Jpn Stand Assoc 70(7):2-31

Wakui Y (2014) Multivariate analysis understanding, Technical commentary, pp 190-200

Yano H (2002) Introduction to quality engineering numeration, Japan Standards Association, pp 271-286

Yano H (2004) Technology development of the information design with the computer-Simulation and MT system, Japan Standards Association, pp 310-368

Yano H (2011) Quality engineering guide to raise an engineer power, Japan Standards Association, pp 219-235 